\title{
Inappropriate antidiuretic hormone secretion associated with bronchial carcinoma, intracranial trauma and hypothyroidism: a diagnostic problem
}

\author{
William J. MacLenNan* \\ M.B., M.R.C.P.(Glasg., Lond.) \\ Lecturer, Department of Materia Medica, University of Glasgow
}

IT Is well recognized that a bronchial carcinoma may be occasionally responsible for a syndrome associated with the inappropriate secretion of antidiuretic hormone (ADH) (Bartter \& Schwartz, 1967). A similar water and electrolyte imbalance has been encountered in patients suffering from myxoedema or from various intracranial lesions (Haden \& Knox, 1965; Neilson \& Thorn, 1965). This report describes a patient in which excessive production of $\mathrm{ADH}$ was associated with all three lesions.

\section{Case report}

D.M., a retired labourer, aged 67 , was admitted to Stobhill General Hospital on 4 April 1968. His behaviour had become strange over the previous 4 weeks. This included invitations to women passing his window to come and see him. Relatives had noted a slight change in his personality ever since a car accident in January 1967. At that time he had suffered from severe concussion, and an X-ray had demonstrated a fracture of the left parietal bone. He had been kept under observation in hospital for 2 weeks. The main symptom after discharge had been recurrent left temporal headaches.

Enquiry about his general health revealed that he had been troubled by occasional bouts of epigastric pain for many years. These usually came on about $2 \mathrm{hr}$ after a meal, and sometimes woke him at night. They had been in remission over the previous 6 months. He also gave a long history of cough with sputum which was often mucopurulent. Relatives stated that he was a heavy drinker, and that he smoked twenty to thirty cigarettes per day.

There was no gross intellectual impairment, though his memory for recent and past events was poor. During his prolonged stay in hospital there were occasional periods, usually lasting several hours,

*Present address: Senior Registrar, Departments of Geriatric Medicine, Stobhill General Hospital and Western Infirmary, Glasgow. when he became very confused, but he was completely rational most of the time. It was noted that his skin was coarse and dry, that there was early finger clubbing, and that there was a pad of sacral oedema but no ankle swelling. The pulse was regular, $64 / \mathrm{min}$, BP $115 / 90 \mathrm{mmHg}$. JVP normal. No abnormalities were noted on examination of the lungs, abdomen or nervous system.

Investigations. Urine: SG between 1010 and 1020, 800-1300 ml were excreted daily. $\mathrm{Hb} 15 \cdot 1 \mathrm{~g} / 100 \mathrm{ml}$; ESR was $4 \mathrm{~mm} / \mathrm{hr}$. Blood urea was $24 \mathrm{mg} / 100 \mathrm{ml}$; serum potassium $5.0 \mathrm{mEq} / \mathrm{l}$. Serum sodium and chloride fluctuated between 119 and $125 \mathrm{mEq} / \mathrm{l}$, and 80 and $88 \mathrm{mEq} / 1$, respectively. Liver function tests were normal. A chest X-ray was negative, and though there was evidence of an old fracture on the skull X-ray, there was no shift of the calcified pineal body from the mid-line. There was EEG evidence of a structural disorder in the left mid-temporal area. Low voltage complexes were recorded in all twelve leads of an ECG, but there was no evidence of myocardial infarction.

The appearance of the skin, the low pulse rate and the character of the ECG complexes were all suggestive of hypothyroidism. The $24-\mathrm{hr}{ }^{131} \mathrm{I}$ uptake was $8 \%$; the ratio of bound to free ${ }^{131} \mathrm{I}$ at $48 \mathrm{hr}$ was $0.07-0.28 \%$ of the dose per litre; $43 \%$ of the dose was excreted in the urine in $0-8 \mathrm{hr}, 23 \%$ at $8-24 \mathrm{hr}$ and $4 \%$ at $24-48 \mathrm{hr}$. These findings confirmed that the patient was mildly hypothyroid.

The electrolyte disturbance was further investigated. The plasma osmolality was $248 \mathrm{~m}$-osmoles/1, but the urine osmolality normal at $298 \mathrm{~m}$-osmoles/1. $120 \mathrm{mEq}$ of sodium were excreted in the urine over $24 \mathrm{hr}$. Measurement of the blood volume with ${ }^{125}$ I-labelled albumin demonstrated a value of 5.06 litres compared with an estimated normal of 4.65 litres for the patient's height and weight. Plasma cortisol levels, their diurnal variation and their response to Synacthen were all within normal limits. 
These findings suggested that there was inappropriate secretion of $\mathrm{ADH}$ and a search was made for a bronchial carcinoma. Six out of nine specimens of sputum contained cells suggestive of malignancy, but there was no evidence of a tumour at bronchoscopy.

A 24-hr collection of urine was assayed for ADHlike activity. The recorded level of $18 \pm 6 \mathrm{mU}$, while above the normal limit of $10 \mathrm{mU}$ was less than the 25-30 mU necessary to provide conclusive evidence of inappropriate ADH secretion.

Attempts at restricting the subject's fluid intake were unsuccessful, and he was treated with fludrocortisone acetate in a dose of $0.3 \mathrm{mg}$ daily. This had no effect upon the serum electrolyte levels. The mild hypothyroidism was corrected with $l$-sodium thyroxine in a dose of $0.05 \mathrm{mg}$ daily.

Over the next 6 months the patient's mental state fluctuated, but no close relationship was demonstrated with the variations in the serum sodium levels. A close scrutiny was kept on the lung fields. Signs of dullness and diminished air entry were noted at the right base on 28 June 1968. A chest $\mathrm{X}$-ray showed that these were due to collapse in the basal segment of the right lower lobe. Tomography confirmed the presence of a basal opacity, but failed to demonstrate lymphadenopathy or bronchial obstruction. Radiology on 15 October 1968 showed that there was a right pleural effusion with further collapse in the affected segments.

There was a sudden deterioration in the patient's condition on 30 November 1968 . He became very confused and started to vomit profusely. The skin was cold and clammy, and the pulse was thready at a rate of 80 beats $/ \mathrm{min}$. The blood pressure had fallen to $95 / 60 \mathrm{mmHg}$. Guarding and tenderness were noted extending from the epigastrium and right hypochondrium to the right iliac fossa. It was thought that deterioration had been due to perforation of a peptic ulcer. Surgery was not performed because the patient was also suffering from malignant disease. Deterioration continued and death occurred on 4 December 1968.

\section{Necropsy}

A bronchial carcinoma about $10 \mathrm{~cm}$ in diameter was found in association with consolidation and collapse of the posterior and lateral segments in the right lower lobe. There was an old infarction in the posterior wall of the left ventricle. The peritoneal cavity was filled with a turbid fluid, and there was evidence of an early generalized peritonitis. This was evidently the result of a perforated peptic ulcer situated on the superior border of the pylorus. No gross abnormalities were noted on inspection of the brain, kidneys or thyroid gland. The tumour consisted mainly of small round cells with scattered clumps of oat cells. The histological appearances of the adrenals, kidneys and thyroid were normal. A section of the tumour was assayed for ADH-like activity. This was found to be $100 \mu \mathrm{U} / \mathrm{mg}$ of dry tissue compared with a normal value of less than $4 \mu \mathrm{U}$.

\section{Discussion}

The diagnostic features of inappropriate secretion of $\mathrm{ADH}$ are hyponatraemia and hyposmolality of the plasma; a normal or high renal excretion of sodium producing urine with a normal or high osmolality; the absence of clinical signs indicating fluid depletion; and normal renal and adrenal function (Bartter \& Schwartz, 1967). All these criteria were established in the patient presented above.

Symptoms and signs of sodium depletion include personality changes, irritability and confusion. Muscle weakness, diminished tendon reflexes and upgoing plantar responses may also be encountered (Bartter \& Schwartz, 1967). It is uncertain, in this particular case, whether the mental disturbance was due to the electrolyte imbalance or to the old head injury. The normal appearance of the brain at necropsy, and the episodic nature of the confusion, favour the former hypothesis.

The results obtained from assay of ADH-like activity in the urine were equivocal, but the other laboratory findings made the diagnosis of inappropriate ADH secretion fairly certain. It was more difficult to define the lesion causing the syndrome. A variety of intracerebral conditions, including trauma, may cause excessive ADH production by the posterior pituitary. This may be due to a disturbance of regulatory centres in the hypothalamus (Haden \& Knox, 1965). The focal signs of the EEG, coupled with the history of head injury, suggested this explanation, but the negative necropsy findings now make this seem less probable. Inappropriate ADH secretion may occur with myxoedema, and can be corrected, in this situation, by the administration of $l$-sodium thyroxine. The physiological basis of these observations is obscure (Nielsen \& Thorn, 1965). There was clinical and laboratory evidence of myxoedema in the case under discussion, but the histological appearance of the thyroid gland was normal. It is possible that the electrolyte disturbance could have caused the thyroid dysfunction. Barraclough, Jones \& Lee (1966) mention that patients with an ADH-secreting tumour often exhibit hypothermia and sluggish tendon reflexes. In view of the large quantity of hormone found in the extract, it is likely that the bronchial carcinoma was the source of ADH-like activity. The tumour, if such is the case, may have been producing large quantities of hormone long before it had manifested itself 
clinically, radiologically or on bronchoscopy. It is well recognized that a bronchial carcinoma can produce humoral effects well in advance of local manifestations (Barraclough et al., 1966).

Various bio-assay techniques have been devised which demonstrate high concentrations of an ADHlike substance in oat cell tumours associated with electrolyte disturbances. Its pharmacological properties resemble those of arginine-vasopressin (Bower, Mason \& Forsham, 1964; Lee, Jones \& Barraclough, 1964; de Souza, Delaere \& Berde, 1965; Barraclough et al., 1966; Godley \& Locke, 1968). Other investigators have demonstrated immunological similarities between the tumour extract and vasopressin (Verherr et al., 1968). These studies suggest that the tumour actually produces ADH. The alternative, that the cancer merely concentrates material secreted by the pituitary, seems less likely.

A reduction in the urinary output caused by continuous hypersecretion of ADH produces a marked increase in volume of the total body water. The most marked increase is in the intracellular compartment, though there is also a slight expansion of fluid in the plasma and interstitial spaces. This is associated with an influx of sodium into the cells, at the expense of the extracellular compartment (Grantham, Brown \& Schloerb, 1965; Kaye, 1966). The excretion of normal or increased quantities of sodium in the presence of hyponatraemia is related to an inhibition of sodium reabsorption by the proximal renal tubules. This would appear to be a direct response by the tubules to an expansion in the extracellular fluid volume (Bartter \& Schwartz, 1967).

An improvement in symptoms, and correction of the electrolyte disturbance, may be achieved by restricting the fluid intake (Thorn \& Transbøl, 1963). It was not possible to keep the patient on this regimen. Saline infusions are of no value since the excess sodium is immediately excreted (Bartter \& Schwartz, 1967). Fludrocortisone reduces sodium excretion if given in large doses, but it does not solve the basic problem of water retention (Ross, 1963). Physiological doses of fludrocortisone may cause a paradoxical increase in sodium loss (Ivy, 1961). A direct attack on the tumour by surgery, radiotherapy or cytotoxic therapy can be extremely effective in this syndrome (Ivy, 1961; Godley \& Locke, 1968; Thorn \& Transbøl, 1968). This was not attempted in the case discussed because of early uncertainty over the diagnosis of bronchial cardinoma, and the possibility of the electrolyte disturbance being related to hypothyroidism or intracranial tumour.

\section{Acknowledgments}

I should like to thank Dr J. Lee of Charing Cross Hospital for performing assays of ADH-like activity on the urine and tumour specimens. I am grateful to Professor Alstead and Dr J. G. Macarthur for their help and encouragement in the preparation of this paper.

\section{References}

Barraclough, M.A., Jones, J.J. \& Lee, J. (1966) Production of vasopressin by anaplastic oat cell carcinoma of the bronchus. Clinical Science, 31, 135.

BARTTER, F.C. \& SchWARTZ, W.B. (1967) The syndrome of inappropriate secretion of antidiuretic hormone. American Journal of Medicine, 42, 790.

Bower, B.F., Mason, D.M. \& Forsham, P.H. (1964) Bronchogenic carcinoma with inappropriate antidiuretic activity in plasma and tumor. New England Journal of Medicine, 271, 934.

GODLEY, M.L. \& LOCKE, W. (1968) Abnormal antidiuresis in bronchogenic carcinoma. American Journal of Surgery, 115, 413.

Grantham, J.J., Brown, R.W. \& Schloerb, P.R. (1965) Asymptomatic hyponatraemia and bronchogenic carcinoma: the deleterious effect of diuretics. American Journal of Medical Science, 249, 273.

HADEN, H.T. \& KNOX, G.W. (1965) Cerebral hyponatraemia with inappropriate antidiuretic hormone secretion.American Journal of Medical Science, 249, 381.

Ivy, H.K. (1961) Renal sodium loss and bronchogenic carcinoma. Associated autonomic neuropathy. Archives of Internal Medicine, 108, 47.

KAYE, M. (1966) An investigation into the cause of hyponatraemia in the syndrome of inappropriate secretion of antidiuretic hormone. American Journal of Medicine, 41, 910.

LeE, J., Jones, J.J. \& Barraclough, M.A. (1964) Inappropriate secretion of vasopressin. Lancet, ii, 792.

NIELSEN, B. \& THORN, N.A. (1965) Inappropriate secretion of antidiuretic hormone due to myxoedema. New England Journal of Medicine, 272, 362.

Ross, E.J. (1963) Hyponatraemic syndromes associated with carcinoma of the bronchus. Quarterly Journal of Medicine, 32, 297.

De Souza, R.C., Delaere, J. \& Berde, B. (1965) Inappropriate secretion of vasopressin. Lancet, $\mathbf{i}, 436$.

ThORN, N.A. \& TransBøl (1963) Hyponatraemia and bronchogenic carcinoma associated with renal excretion of large amounts of antidiuretic material. American Journal of Medicine, 35, 257.

VerherR, H., MASSRY, S.G., UTIGER, R.D. \& KLEMON, C.R. (1968) Antidiuretic principle in malignant tumor extracts from patients with inappropriate ADH syndrome. Journal of Clinical Endocrinology, 28, 162. 